\title{
Reliability-Centered Maintenance: Analyzing Failure in Harvest Sugarcane Machine Using Some Generalizations of the Weibull Distribution
}

\author{
Pedro L. Ramos (D), Diego C. Nascimento, Camila Cocolo, Márcio J. Nicola, \\ Carlos Alonso, Luiz G. Ribeiro, André Ennes, and Francisco Louzada $\mathbb{D}$ \\ Institute of Mathematical and Computer Sciences, São Paulo University, São Carlos, SP, Brazil \\ Correspondence should be addressed to Pedro L. Ramos; pedrolramos@usp.br
}

Received 7 December 2017; Revised 22 January 2018; Accepted 14 February 2018; Published 1 April 2018

Academic Editor: Farouk Yalaoui

Copyright (C) 2018 Pedro L. Ramos et al. This is an open access article distributed under the Creative Commons Attribution License, which permits unrestricted use, distribution, and reproduction in any medium, provided the original work is properly cited.

\begin{abstract}
We considered five generalizations of the standard Weibull distribution to describe the lifetime of two important components of sugarcane harvesting machines. The harvesters considered in the analysis harvest an average of 20 tons of sugarcane per hour and their malfunction may lead to major losses; therefore, an effective maintenance approach is of main interest for cost savings. For the considered distributions, mathematical background is presented. Maximum likelihood is used for parameter estimation. Further, different discrimination procedures were used to obtain the best fit for each component. At the end, we propose a maintenance scheduling for the components of the harvesters using predictive analysis.
\end{abstract}

\section{Introduction}

The arrival of the sugarcane culture in Brazil has had a significant impact on the national economy, which led the country to become the largest producer in the world [1]. Its subproducts are used in the food and chemical industries, as well as in electricity generation and fuel production. Mechanized harvesting is one of the most important stages in the sugar and ethanol mills, since it provides the raw material with quality, time, and competitive costs for later processing. Among the used machines in the mechanized harvest, the harvesters stand out for having a large number of corrective stops, given the functionality in such extreme environmental conditions. In addition, its operation is in a regime of 24 hours on the workdays, having impact on fatigue and wear of their parts. During operation, the harvester processes an average of 20 tons of sugarcane per hour and its malfunction may lead to major losses; therefore, an effective maintenance approach is of keen interest [2].

Reliability-centered maintenance consists of determining the most effective maintenance approach $[3,4]$. This process was firstly developed in the aviation industry for deciding what maintenance work is needed to keep aircraft airborne, driven by the need to improve reliability, while reducing the cost of maintenance [5]. Reliability analysis can be used to estimate time-related parameters to the next machine stop [6], providing information to manage and control the preventive maintenance of harvesters which could result in increased production and has potential for cost savings.

In reliability, common procedures are usually based on the assumption that the data follows a Weibull distribution. Introduced by Weibull [7], this distribution has convenient mathematical properties and its physiological failure process arises in many areas (see Manton and Yashin [8]). Additionally, McCool [9] provided an extensive discussion about its use in reliability. However, this distribution cannot be used to describe data with nonmonotone hazard function (bathtub, upside-down bathtub, to list a few). To overcome this problem, many generalizations of the standard Weibull distribution have been proposed. Murthy et al. [10] presented the application of some generalized Weibull distributions for modeling complex failure data sets. Pham and Lai [11] discussed recent generations of Weibull-related lifetime. Further, Lai [12] reviewed more than 25 generalizations of the Weibull distribution, and Tahir and Cordeiro [13] cited more than 30 compounded Weibull models. 
In this paper, we consider five important generalized Weibull distributions with three parameters to describe the lifetime of two important components of the sugarcane harvesting machines. Our main goal here is to correctly predict the next failure of the components, not to present an extensive review of the generalizations of Weibull distribution. In the cited papers, the authors only described mathematical properties of the distributions and conducted the fit for different data. However, reliability is more about correctly predicting the future than describing the past; in this sense, no predictive analysis was presented considering such generalizations.

The distributions considered are the gamma-Weibull distribution [14], generalized Weibull (GW) distribution [15], exponentiated Weibull (EW) distribution [16], Marshall-Olkin Weibull (MOW) distribution [17], and the extended PoissonWeibull (EPW) distribution [18]. While the first three distributions are the most common three parameter generalization of the Weibull, the MOW and the EPW arise in the competitive and complementary risk scenario (see Louzada [19], for a detailed discussion). In these cases, the latent variables follow, respectively, a geometric and a zero-truncated Poisson distribution and each of components in risk came from a Weibull baseline distribution.

For each distribution, the mathematical background is reviewed and the parameters estimators are presented using the maximum likelihood estimators. Further, different discrimination procedures are used to obtain the best fit for each component. At the end, we propose a maintenance scheduling for the components of the harvesters using predictive analysis.

The remainder of this paper is organized as follows. Section 2 presents the literature review related to the survival models adopted. Section 3 exposes the data collection and empirical analysis, as well as carrying out the predictive analysis based on the parametric models. Finally, in Section 4, we present some final remarks related to the contribution of this study.

\section{Theoretical Background}

In this section, we present the statistical background on the adopted distributions and its parameter estimation procedures. The following distributions are considered: gamma-Weibull, generalized Weibull, exponentiated Weibull, Marshall-OlkinWeibull, and Marshall-Olkin-Weibull. Their choice is based on their flexibility to accommodate lifetime dataset with hazard functions with different shapes, for instance, constant, increasing, decreasing, bathtub, and upside-down bathtub.

2.1. The Gamma-Weibull Distribution. Introduced by Stacy [14], the gamma-Weibull distribution with three parameters is a flexible model for reliability data due to its ability to accommodate various forms of the hazard function. This distribution is also known as generalized gamma (GG) distribution as it generalizes the two-parameter gamma distribution; hereafter, we will refer to this model as GG distribution to avoid confusion with the GW distribution. A random variable has GG distribution if its probability density function $(\mathrm{PDF})$ is given by

$$
f(t ; \phi, \mu, \alpha)=\frac{\alpha}{\Gamma(\phi)} \mu^{\alpha \phi} t^{\alpha \phi-1} e^{-(\mu t)^{\alpha}}, \quad t>0
$$

where $\alpha>0, \phi>0$, and $\mu>0$. The mean and variance of GG are given by

$$
\begin{aligned}
E(X) & =\frac{\Gamma(\phi+1 / \alpha)}{\mu \Gamma(\phi)}, \\
V(X) & =\frac{1}{\mu^{2}}\left\{\frac{\Gamma(\phi+2 / \alpha)}{\Gamma(\phi)}-\left(\frac{\Gamma(\phi+1 / \alpha)}{\Gamma(\phi)}\right)^{2}\right\} .
\end{aligned}
$$

Some relevant distributions are special cases such as the Weibull distribution (when $\phi=1$ ), the distribution gamma $(\alpha=1)$, log-normal (case limit when $\phi \rightarrow \infty)$, and the generalized normal distribution $(\alpha=2)$. For example, the generalized normal distribution is also a distribution that includes several distributions known as half-normal $(\phi=1 / 2, \mu=$ $1 / \sqrt{2} \sigma)$, Rayleigh $(\phi=1, \mu=1 / \sqrt{2} \sigma)$, Maxwell-Boltzmann $(\phi=3 / 2)$ and chi $(\phi=k / 2, k=1,2, \ldots)$. The cumulative distribution function (CDF) is given by

$$
F(t ; \phi, \mu, \alpha)=\int_{0}^{(\mu t)^{\alpha}} \frac{1}{\Gamma(\phi)} w^{\phi-1} e^{-w} d w=\frac{\gamma\left[\phi,(\mu t)^{\alpha}\right]}{\Gamma(\phi)},
$$

where $\gamma[y, x]=\int_{0}^{x} w^{y-1} e^{-w} d w$ is the lower incomplete gamma function. The survival function is

$$
S(t ; \phi, \mu, \alpha)=1-F(t ; \phi, \mu, \alpha)=\frac{\Gamma\left[\phi,(\mu t)^{\alpha}\right]}{\Gamma(\phi)},
$$

where $\Gamma[y, x]=\int_{x}^{\infty} w^{y-1} e^{-w} d w$ is the upper incomplete gamma function.

The hazard function of the GG distribution is

$$
\begin{aligned}
h(t ; \phi, \mu, \alpha) & =\frac{f(t ; \phi, \mu, \alpha)}{S(t ; \phi, \mu, \alpha)} \\
& =\frac{\alpha \mu^{\alpha \phi} t^{\alpha \phi-1} \exp \left(-(\mu t)^{\alpha}\right)}{\Gamma\left[\phi,(\mu t)^{\alpha}\right]},
\end{aligned}
$$

where the hazard function has constant, increasing, decreasing, bathtub, and upside-down bathtub hazard rate.

For parameter estimation, let $T_{1}, \ldots, T_{n}$ be a random sample of size $n$, where $T \sim \mathrm{GG}(\alpha, \mu, \phi)$. Then, the likelihood function related to the PDF (1) is given by

$$
\begin{aligned}
& L(\phi, \mu, \alpha ; \mathbf{t}) \\
& \quad=\frac{\alpha^{n}}{\Gamma(\phi)^{n}} \mu^{n \alpha \phi}\left\{\prod_{i=1}^{n} t_{i}^{\alpha \phi-1}\right\} \exp \left\{-\mu^{\alpha} \sum_{i=1}^{n} t_{i}^{\alpha}\right\} .
\end{aligned}
$$

The log-likelihood is given by

$$
\begin{aligned}
l(\phi, \mu, \alpha ; \mathbf{t})= & n \log (\alpha)-n \log \Gamma(\phi)+n \alpha \phi \log (\mu) \\
& +(\alpha \phi-1) \sum_{i=1}^{n} \log \left(t_{i}\right)-\mu^{\alpha} \sum_{i=1}^{n} t_{i}^{\alpha} .
\end{aligned}
$$


Setting the partial derivatives $(\partial / \partial \alpha) l(\phi, \mu, \alpha ; \mathbf{t}), \quad(\partial /$ $\partial \mu) l(\phi, \mu, \alpha ; \mathbf{t})$, and $(\partial / \partial \phi) l(\phi, \mu, \alpha ; \mathbf{t})$ equal to 0 , we obtain the following maximum likelihood estimators:

$$
\begin{aligned}
& \widehat{\mu}=\left(\frac{1}{\widehat{\alpha}}\right. \\
&\left.\cdot \frac{n}{\sum_{i=1}^{n} t_{i}^{\widehat{\alpha}} \log \left(t_{i}\right)-\left(\left(\sum_{i=1}^{n} t_{i}^{\widehat{\alpha}}\right) / n\right) \sum_{i=1}^{n} \log \left(t_{i}\right)}\right)^{1 / \alpha}, \\
& \widehat{\phi}=\left(\frac{1}{\widehat{\alpha}} \frac{\sum_{i=1}^{n} t_{i}^{\widehat{\alpha}}}{\sum_{i=1}^{n} t_{i}^{\widehat{\alpha}} \log \left(t_{i}\right)-\left(\left(\sum_{i=1}^{n} t_{i}^{\widehat{\alpha}}\right) / n\right) \sum_{i=1}^{n} \log \left(t_{i}\right)}\right), \\
& n \widehat{\alpha} \log (\widehat{\mu})+\widehat{\alpha} \sum_{i=1}^{n} \log \left(t_{i}\right)-n \psi(\widehat{\phi})=0,
\end{aligned}
$$

where $\psi(k)=(\partial / \partial k) \log \Gamma(k)=\Gamma^{\prime}(k) / \Gamma(k)$. The solution provides the maximum likelihood estimates (MLEs). See, for instance, Ramos et al. [20, 21] and Achcar et al. [22] for a detailed discussion.

Under mild conditions, the estimators become unbiased for large samples and asymptotically efficient. Moreover, such estimators have asymptotically normal joint distribution given by

$$
(\widehat{\phi}, \widehat{\mu}, \widehat{\alpha}) \sim N_{3}\left[(\phi, \mu, \alpha), I^{-1}(\phi, \mu, \alpha)\right] \quad \text { for } n \longrightarrow \infty,
$$

where $I(\boldsymbol{\theta})$ is the Fisher information matrix: that is,

$$
\left[\begin{array}{ccc}
\frac{1+2 \psi(\phi)+\phi \psi^{\prime}(\phi)+\phi \psi(\phi)^{2}}{\alpha^{2}} & -\frac{1+\phi \psi(\phi)}{\mu} & -\frac{\psi(\phi)}{\alpha} \\
-\frac{1+\phi \psi(\phi)}{\mu} & \frac{\phi \alpha^{2}}{\mu^{2}} & \frac{\alpha}{\mu} \\
-\frac{\psi(\phi)}{\alpha} & \frac{\alpha}{\mu} & \psi^{\prime}(\phi)
\end{array}\right]
$$

and $\psi^{\prime}(k)=(\partial / \partial k) \psi(k)$ is the trigamma function.

2.2. The Generalized Weibull Distribution. Introduced by Mudholkar et al. [15], the generalized Weibull distribution has PDF given by

$$
\begin{aligned}
& f(t ; \lambda, \beta, \alpha) \\
& \quad=(\alpha \beta)^{-1}\left(\frac{t}{\beta}\right)^{1 / \alpha-1}\left(1-\lambda\left(\frac{t}{\beta}\right)^{1 / \alpha}\right)^{1 / \lambda-1},
\end{aligned}
$$

where $\lambda \in \mathbb{R}, \beta>0$, and $\alpha>0$. The CDF and the survival function are, respectively, given by

$$
\begin{aligned}
& F(t ; \lambda, \beta, \alpha)=1-\left(1-\lambda\left(\frac{t}{\beta}\right)^{1 / \alpha}\right)^{1 / \lambda}, \\
& S(t ; \lambda, \beta, \alpha)=\left(1-\lambda\left(\frac{t}{\beta}\right)^{1 / \alpha}\right)^{1 / \lambda} .
\end{aligned}
$$

The hazard function of the GW distribution is

$$
h(t ; \lambda, \beta, \alpha)=\frac{(t / \beta)^{1 / \alpha-1}}{\alpha \beta\left(1-\lambda(t / \beta)^{1 / \alpha}\right)} .
$$

This model is very flexible to describe lifetime data, since it has the hazard function with constant, increasing, decreasing, bathtub, and upside-down bathtub hazard rate. The quantile function of the GW distribution has closed form and is given by

$$
Q(u ; \lambda, \beta, \alpha)= \begin{cases}\beta(-\log (1-u))^{\alpha} & \text { if } \lambda=0, \\ \beta\left(\frac{1-(1-u)^{\lambda}}{\lambda}\right)^{\alpha} & \text { if } \lambda \neq 0 .\end{cases}
$$

For parameter estimation, let $T_{1}, \ldots, T_{n}$ be a random sample of size $n$, where $T \sim \mathrm{GW}(\alpha, \mu, \beta)$. Then, the likelihood function related to the PDF (11) is given by

$$
\begin{aligned}
& L(\lambda, \beta, \alpha ; \mathbf{t}) \\
& \quad=(\alpha \beta)^{-n} \prod_{i=1}^{n}\left(\frac{t_{i}}{\beta}\right)^{1 / \alpha-1}\left(1-\lambda\left(\frac{t_{i}}{\beta}\right)^{1 / \alpha}\right)^{1 / \lambda-1} .
\end{aligned}
$$

The log-likelihood is given by

$$
\begin{aligned}
l(\lambda, \beta, \alpha ; \mathbf{t})= & \left(\frac{1}{\lambda}-1\right) \sum_{i=1}^{n} \log \left(1-\lambda\left(\frac{t_{i}}{\beta}\right)^{1 / \alpha}\right) \\
& -n \log (\alpha \beta)+\left(\frac{1}{\alpha}-1\right) \sum_{i=1}^{n} \log \left(\frac{t_{i}}{\beta}\right) .
\end{aligned}
$$

Setting the partial derivatives equal to 0 , we obtain the maximum likelihood estimators. Here, we follow Mudholkar et al. [15] which considers the direct maximization of (16). Under mild conditions, the obtained estimators are consistent and efficient with an asymptotically normal joint distribution given by

$$
\widehat{\Theta} \sim N_{3}\left[\Theta, I^{-1}(\Theta)\right] \quad \text { as } n \longrightarrow \infty,
$$

where $I(\Theta)$ is the $3 \times 3$ Fisher information matrix associated with the vector of parameters $\Theta$ and $I_{i j}(\Theta)$ is the Fisher information elements in $i$ and $j$ given by

$$
I_{i j}(\Theta)=E\left[-\frac{\partial^{2}}{\partial \Theta_{i} \partial \Theta_{j}} l(\Theta ; \mathscr{D})^{2}\right], \quad i, j=1,2,3 .
$$

Since the Fisher information matrix does not have closedform expression for some terms, an alternative is to consider the observed information matrix, where the terms are given by

$$
H_{i j}(\Theta)=-\frac{\partial^{2}}{\partial \Theta_{i} \partial \Theta_{j}} l(\Theta ; \mathbf{t})^{2}, \quad i, j=1,2,3 .
$$

Hereafter, we considered the same approach to obtain the confidence intervals for the parameters from other distributions. 
2.3. The Exponentiated Weibull Distribution. Introduced by Mudholkar et al. [16], the exponentiated Weibull distribution with $\mathrm{PDF}$ is given by

$$
\begin{aligned}
f(t ; \sigma, \phi, \alpha)= & \alpha \frac{\phi}{\sigma}\left(\frac{t}{\sigma}\right)^{\alpha-1} \exp \left(-\left(\frac{t}{\sigma}\right)^{\alpha}\right) \\
& \cdot\left(1-\exp \left(-\left(\frac{t}{\sigma}\right)^{\alpha}\right)\right)^{\phi-1},
\end{aligned}
$$

where $\sigma>0, \phi>0$, and $\alpha>0$.

The exponentiated Weibull distribution includes the Weibull distribution $(\phi=1)$ and the exponentiated exponential distribution $(\alpha=1)$. The survival function is given by

$$
S(t ; \sigma, \phi, \alpha)=1-\left(1-\exp \left(-\left(\frac{t}{\sigma}\right)^{\alpha}\right)\right)^{\phi} .
$$

The hazard function of the GG distribution is

$$
\begin{aligned}
& h(t ; \phi, \mu, \alpha) \\
& =\frac{\alpha \phi(t / \sigma)^{\alpha-1} \exp \left(-(t / \sigma)^{\alpha}\right)\left(1-\exp \left(-(t / \sigma)^{\alpha}\right)\right)^{\phi-1}}{\sigma\left(1-\left(1-\exp \left(-(t / \sigma)^{\alpha}\right)\right)^{\phi}\right)} .
\end{aligned}
$$

The shapes of the hazard function are analogous to the GG and GW distribution. Additionally, the quantile function of the EW distribution has closed form and is given by

$$
Q(u ; \sigma, \phi, \alpha)=\sigma\left(-\log \left(1-u^{1 / \phi}\right)\right)^{1 / \alpha}
$$

The $k$ th moment of the EW distribution is given by

$$
\begin{aligned}
\mu_{k} & =\int_{0}^{1} Q(u ; \sigma, \phi, \alpha)^{k} d u \\
& =\theta \sigma^{k} \Gamma\left(\frac{k}{\alpha}+1\right)\left(1+\sum_{i=1}^{\infty} a_{i}\left[(i+1)^{k / \alpha+1}\right]\right),
\end{aligned}
$$

where $k \in \mathbb{N}, a_{i}=(-1)^{i}(\theta-1)(\theta-2) \cdots(\theta-1-\overline{i-1})(i !)^{-1}$. The proof of this equality is presented by Choudhury [23].

For parameter estimation, let $T_{1}, \ldots, T_{n}$ be a random sample of size $n$, where $T \sim \operatorname{EW}(\sigma, \phi, \alpha)$. Then, the likelihood function related to the PDF (20) is given by

$$
\begin{aligned}
L(\sigma, \phi, \alpha ; \mathbf{t})= & \frac{\alpha^{n} \phi^{n}}{\sigma^{n}} \prod_{i=1}^{n}\left(\frac{t_{i}}{\sigma}\right)^{\alpha-1} \\
& \cdot\left(1-\exp \left(-\left(\frac{t_{i}}{\sigma}\right)^{\alpha}\right)\right)^{\phi-1} \\
& \cdot \exp \left(-\sum_{i=1}^{n}\left(\frac{t_{i}}{\sigma}\right)^{\alpha}\right)
\end{aligned}
$$

The log-likelihood is given by

$$
\begin{aligned}
l(\sigma, \phi, \alpha ; \mathbf{t})= & n \log (\alpha \phi) \\
& +(\phi-1) \sum_{i=1}^{n} \log \left(1-\exp \left(-\left(\frac{t_{i}}{\sigma}\right)^{\alpha}\right)\right) \\
& +(\alpha-1) \sum_{i=1}^{n} \log \left(t_{i}\right)-\sum_{i=1}^{n}\left(\frac{t_{i}}{\sigma}\right)^{\alpha} \\
& -n \alpha \log (\sigma) .
\end{aligned}
$$

Setting the partial derivatives $(\partial / \partial \sigma) l(\sigma, \phi, \alpha ; \mathbf{t}), \quad(\partial /$ $\partial \phi) l(\sigma, \phi, \alpha ; \mathbf{t})$, and $(\partial / \partial \alpha) l(\sigma, \phi, \alpha ; \mathbf{t})$ equal to 0 , we obtain the following maximum likelihood estimators:

$$
\begin{aligned}
& \frac{n}{\alpha}+\sum_{i=1}^{n} \log \left(t_{i}\right)+\frac{(\phi-1)}{\sigma^{\alpha}} \sum_{i=1}^{n} \frac{t_{i}^{\alpha} \log \left(t_{i} / \sigma\right)}{\exp \left(\left(t_{i} / \sigma\right)^{\alpha}\right)-1} \\
& \quad-\sum_{i=1}^{n}\left(\frac{t_{i}}{\sigma}\right)^{\alpha} \log \left(\frac{t_{i}}{\sigma}\right) \exp \left(-\left(\frac{t_{i}}{\sigma}\right)^{\alpha}\right) \\
& \quad-n \log (\sigma)=0, \\
& -\frac{n \alpha}{\sigma}-\frac{\alpha}{\sigma^{\alpha+1}} \sum_{i=1}^{n} t_{i}^{\alpha}+\frac{\alpha}{\sigma^{\alpha}} \sum_{i=1}^{n} \frac{(\phi-1) t_{i}^{\alpha}}{\sigma-\sigma \exp \left(\left(t_{i} / \sigma\right)^{\alpha}\right)}=0 \\
& \phi=-\frac{n}{\sum_{i=1}^{n} \log \left(1-\exp \left(-\left(t_{i} / \sigma\right)^{\alpha}\right)\right)} .
\end{aligned}
$$

2.4. The Marshall-Olkin-Weibull Distribution. Marshall and Olkin [17] presented a new procedure for introducing an additional parameter into a family of distribution. In this case, the authors applied such procedure in the Weibull distribution. The obtained PDF of the MOW distribution is given by

$$
f(t ; \lambda, \alpha, \gamma)=\frac{\alpha \gamma \lambda t^{\gamma-1} e^{-\lambda t^{\gamma}}}{\left(1-(1-\alpha) e^{-\lambda t^{\gamma}}\right)^{2}},
$$

where $\lambda>0, \alpha>0$, and $\gamma>0$. The MOW distribution arises naturally in competing risks scenarios. Let $X=$ $\min \left(T_{1}, T_{2}, \ldots, T_{M}\right)$, where $M$ is a random variable with geometrical distribution and $T_{i}$ are assumed to be independent and identically distributed according to a Weibull distribution; then the $X$ has a PDF given by (28). Cordeiro and Lemonte [24] derived many properties and the parameter estimators for the MOW distribution; the following results were obtained from the cited work. The survival function is given by

$$
S(t ; \lambda, \alpha, \gamma)=1-\frac{1-e^{-\lambda t^{\gamma}}}{1-(1-\alpha) e^{-\lambda t^{\gamma}}}
$$

The hazard function of the MOW distribution is

$$
h(t ; \lambda, \alpha, \gamma)=\frac{\gamma \lambda t^{\gamma-1}}{1-(1-\alpha) e^{-\lambda t^{\gamma}}},
$$


where its behavior is constant, increasing, decreasing, bathtub, and unimodal. Moreover, the quantile function of the MOW distribution has closed form and is given by

$$
Q(u ; \lambda, \alpha, \gamma)=\lambda^{-1 / \gamma}\left(\log \left(\frac{1-(1-\alpha) u}{1-u}\right)\right)^{1 / \gamma}
$$

For parameter estimation, let $T_{1}, \ldots, T_{n}$ be a random sample of size $n$, where $T \sim \operatorname{MOW}(\lambda, \alpha, \gamma)$. Then, the likelihood function related to the PDF (28) is given by

$$
\begin{aligned}
& L(\lambda, \alpha, \gamma ; \mathbf{t}) \\
& \quad=\alpha^{n} \gamma^{n} \lambda^{n} \prod_{i=1}^{n} \frac{t_{i}^{\gamma-1}}{\left(1-(1-\alpha) e^{-\lambda t_{i}^{\gamma}}\right)^{2}} \exp \left(-\lambda \sum_{i=1}^{n} t_{i}^{\gamma}\right)
\end{aligned}
$$

The log-likelihood is given by

$$
\begin{aligned}
l(\lambda, \alpha, \gamma ; \mathbf{t})= & n \log (\alpha)+n \log (\gamma)+n \log (\lambda) \\
& +(\gamma-1) \sum_{i=1}^{n} \log \left(t_{i}\right) \\
& -2 \sum_{i=1}^{n} \log \left(1-(1-\alpha) e^{-\lambda t_{i}^{\gamma}}\right)-\lambda \sum_{i=1}^{n} t_{i}^{\gamma}
\end{aligned}
$$

Setting the partial derivatives $(\partial / \partial \lambda) l(\lambda, \alpha, \gamma ; \mathbf{t}), \quad(\partial /$ $\partial \alpha) l(\lambda, \alpha, \gamma ; \mathbf{t})$, and $(\partial / \partial \gamma) l(\lambda, \alpha, \gamma ; \mathbf{t})$ equal to 0 , we obtain the following maximum likelihood estimators:

$$
\begin{aligned}
& \frac{n}{\alpha}-2 \sum_{i=1}^{n} \frac{e^{-\lambda t_{i}^{\gamma}}}{1-(1-\alpha) e^{-\lambda t_{i}^{\gamma}}}=0, \\
& \frac{n}{\lambda}-\sum_{i=1}^{n} t_{i}^{\gamma}-2(1-\alpha) \sum_{i=1}^{n} \frac{t_{i}^{\gamma} e^{-\lambda t_{i}^{\gamma}}}{1-(1-\alpha) e^{-\lambda t_{i}^{\gamma}}}=0, \\
& \frac{n}{\gamma}+\sum_{i=1}^{n} \log \left(t_{i}\right)-2(1-\alpha) \sum_{i=1}^{n} \frac{\lambda t_{i}^{\gamma} \log \left(t_{i}\right) e^{-\lambda t_{i}^{\gamma}}}{1-(1-\alpha) e^{-\lambda t_{i}^{\gamma}}} \\
& \quad=\lambda \sum_{i=1}^{n} t_{i}^{\gamma} \log \left(t_{i}\right)
\end{aligned}
$$

for more details, see Cordeiro and Lemonte [24].

2.5. The Extended Poisson-Weibull Distribution. Ramos et al. [18] introduced the extended Poisson-Weibull (EPW) distribution as a generalization of Weibull-Poisson distribution (see Hemmati et al. [25]), where its PDF is given by

$$
f(t ; \lambda, \alpha, \phi)=\frac{\alpha \lambda \phi t^{\alpha-1} e^{-\phi t^{\alpha}-\lambda e^{-\phi t^{\alpha}}}}{1-e^{-\lambda}}
$$

where $\lambda \in \mathbb{R}^{*}, \phi>0$, and $\alpha>0$. Analogously to the MOW distribution, the EWP model arises naturally in competing risks scenarios. Let $X=\min \left(T_{1}, T_{2}, \ldots, T_{M}\right)$, where $M$ is a random variable with a zero-truncated Poisson distribution and $T_{i}$ are assumed to be independent and identically distributed according to a Weibull distribution, then the $X$ has a $\mathrm{PDF}$ given by (35). The survival function is given by

$$
S(t ; \lambda, \alpha, \phi)=\frac{1-\exp \left(-\lambda e^{-\phi t^{\alpha}}\right)}{1-e^{-\lambda}} .
$$

The hazard function of the GG distribution is

$$
h(t ; \lambda, \alpha, \phi)=\lambda \phi t^{\alpha-1} e^{-\phi t^{\alpha}-\lambda e^{-\phi t^{\alpha}}}\left(1-e^{-\lambda e^{-\phi t^{\alpha}}}\right)^{-1} \text {. }
$$

For the EWP distribution, the hazard function has different shapes such as constant, increasing, decreasing, bathtub, and upside-down bathtub. Furthermore, the quantile function of the EPW distribution has closed form and is given by

$$
\begin{aligned}
Q & (u ; \lambda, \alpha, \phi) \\
& =\left(-\frac{1}{\phi} \log \left(1-\frac{\log \left(\left(e^{\lambda}-1\right) p+1\right)}{\lambda}\right)\right)^{1 / \alpha} .
\end{aligned}
$$

For parameter estimation, let $T_{1}, \ldots, T_{n}$ be a random sample of size $n$, where $T \sim \operatorname{EPW}(\lambda, \alpha, \phi)$. Then, the likelihood function related to the PDF (20) is given by

$$
\begin{aligned}
& L(\lambda, \alpha, \phi ; \mathbf{t}) \\
& \quad=\frac{\alpha^{n} \lambda^{n} \phi^{n}}{\left(1-e^{-\lambda}\right)^{n}} \prod_{i=1}^{n} t_{i}^{\alpha-1} \exp \left(-\phi \sum_{i=1}^{n} t_{i}^{\alpha}-\lambda \sum_{i=1}^{n} e^{-\phi t_{i}^{\alpha}}\right) .
\end{aligned}
$$

The log-likelihood is given by

$$
\begin{aligned}
l(\lambda, \alpha, \phi ; \mathbf{t})= & n \log (\alpha \lambda \phi)-n \log \left(1-e^{-\lambda}\right)-\phi \sum_{i=1}^{n} t_{i}^{\alpha} \\
& +(\alpha-1) \sum_{i=1}^{n} \log \left(t_{i}\right)-\lambda \sum_{i=1}^{n} e^{-\phi t_{i}^{\alpha}} .
\end{aligned}
$$

Setting the partial derivatives $(\partial / \partial \lambda) l(\lambda, \alpha, \phi ; \mathbf{t}), \quad(\partial /$ $\partial \alpha) l(\lambda, \alpha, \phi ; \mathbf{t})$, and $(\partial / \partial \phi) l(\lambda, \alpha, \phi ; \mathbf{t})$ equal to 0 , we obtain the following maximum likelihood estimators:

$$
\begin{aligned}
& \frac{n}{\lambda}+\frac{n}{1-e^{\lambda}}-\sum_{i=1}^{n} e^{-\phi t_{i}^{\alpha}}=0 \\
& \frac{n}{\alpha}+\sum_{i=1}^{n} \log \left(t_{i}\right)-\phi \sum_{i=1}^{n} t_{i}^{\alpha} \log \left(t_{i}\right)+\phi \lambda \sum_{i=1}^{n} t_{i}^{\alpha} \log \left(t_{i}\right) e^{-\phi t_{i}^{\alpha}} \\
& \quad=0 \\
& \frac{n}{\phi}-\sum_{i=1}^{n} t_{i}^{\alpha}+\lambda \sum_{i=1}^{n} t_{i}^{\alpha} e^{-\phi t_{i}^{\alpha}}=0
\end{aligned}
$$

2.6. Goodness of Fit. Firstly, in order to verify the behavior of the empirical data, the Total Time on Test plot (TTT-plot) was considered (Barlow and Campo [26]). The TTT-plot is obtained through the plot of $[r / n, G(r / n)]$, where

$$
G\left(\frac{r}{n}\right)=\frac{\left(\sum_{i=1}^{r} t_{i}+(n-r) t_{(r)}\right)}{\sum_{i=1}^{n} t_{i}},
$$


TABLE 1: Maintenance distribution preventive $(\mathrm{P})$ and corrective $(\mathrm{C})$ stops per crop.

\begin{tabular}{lcccccc}
\hline & \multicolumn{2}{c}{ Crop 1 } & \multicolumn{2}{c}{ Crop 2 } & \multicolumn{2}{c}{ Crop 3 } \\
& P & C & P & C & P & C \\
\hline Machine A & 39 & 232 & 32 & 255 & 23 & 127 \\
Machine B & 37 & 199 & 32 & 182 & 23 & 166 \\
\hline
\end{tabular}

$r=1, \ldots, n, i=1, \ldots, n$, and $t_{(i)}$ is the ordered data. For data with concave (convex) curve, the hazard function has increasing (decreasing) shape. If the behavior starts convex and then becomes concave (concave and then convex), the hazard function has bathtub (inverse bathtub) shape.

The goodness of fit is checked considering the Kolmogorov-Smirnov (KS) test. This procedure is based on the KS statistic $D_{n}=\sup \left|F_{n}(t)-F(t ; \boldsymbol{\theta})\right|$, where sup $t$ is the supremum of the set of distances, $F_{n}(t)$ is the empirical distribution function, and $F(t ; \boldsymbol{\theta})$ is CDF. A hypothesis test is conducted at the $5 \%$ level of significance to test whether or not the data comes from $F(t ; \boldsymbol{\theta})$. In this case, the null hypothesis is rejected if the returned $p$ value is smaller than 0.05 .

The following discrimination criterion methods were adopted: Akaike information criteria (AIC) and the corrected AIC (AICc) computed, respectively, by AIC $=-2 l(\widehat{\boldsymbol{\theta}} ; \mathbf{t})+2 k$ and $\mathrm{AICc}=\mathrm{AIC}+2 k(k+1)(n-k-1)^{-1}$, where $k$ is the number of parameters to be fitted and $\widehat{\boldsymbol{\theta}}$ is MLEs of $\boldsymbol{\theta}$. For a set of candidate models for $\mathbf{t}$, the best one provides the minimum values.

\section{Data Collection and Empirical Analysis}

The dataset came from two sources: a manual stop system, which brings the history of revisions and corrective stops of two sugarcane harvesters; and data from the onboard computers of the harvesters, which provide information on the operation of the machine. The data were collected from January 2015 to August 2017, a period corresponding to 2.5 harvests (crops), that is, a period of thirty months of activity.

3.1. Empirical Analysis. Firstly, considering all the stops and their reasons, records of the performance of the predictive maintenance are required to be observed. In total, 1347 stops were observed, of which 186 were preventive and 1161 corrective stops. Thus, it is possible to observe the superior amount of unplanned stops, thus questioning the effectiveness of preventive maintenance. Table 1 shows the failure among the harvests, considering both machines analysis.

The Pricker and transmission from each machine were selected given their complexity in the maintenance. Figure 1 describes the number of failures per year divided by harvest, considering their temporal sparsity, by which items analyzed in this report correspond to $18 \%$ of the stops.

It is possible to notice a difference in the machines' behavior; both machines appear to be equally affected by the problems of transmission and Pricker, but the machine $B$ is
TABLE 2: Dataset related to the sugarcane harvester's Pricker.

\begin{tabular}{lccccccccccc}
\hline 1 & 1 & 1 & 1 & 1 & 1 & 1 & 1 & 2 & 2 & 2 & 2 \\
2 & 3 & 3 & 3 & 3 & 3 & 4 & 4 & 4 & 5 & 5 & 5 \\
6 & 6 & 7 & 8 & 9 & 11 & 11 & 12 & 14 & 16 & 18 & 18 \\
18 & 22 & 22 & 23 & 29 & 32 & 34 & 38 & 41 & 46 & 53 & 53 \\
\hline
\end{tabular}

TABLE 3: Results of AIC and AICc criteria and the $p$ value from the KS test for all fitted distributions considering the Pricker A.

\begin{tabular}{lccccc}
\hline Criteria & GG & GW & EW & MOW & EPW \\
\hline AIC & 341.013 & 343.696 & $\mathbf{3 4 0 . 4 3 5}$ & 341.317 & 342.750 \\
AICc & 335.559 & 338.241 & $\mathbf{3 3 4 . 9 8 1}$ & 335.862 & 343.296 \\
KS & 0.6735 & 0.6046 & 0.7447 & 0.7457 & 0.5751 \\
\hline
\end{tabular}

TABLE 4: MLEs, standard deviations, and 95\% confidence intervals for $\alpha, \theta, \sigma$, and $y^{*}$ related to the EW distribution.

\begin{tabular}{lccc}
\hline $\boldsymbol{\theta}$ & MLE & $\mathrm{SD}$ & $\mathrm{CI}_{95 \%}(\boldsymbol{\theta})$ \\
\hline$\alpha$ & 0.379 & 0.044 & $(0.3181 ; 0.4969)$ \\
$\theta$ & 6.446 & 0.703 & $(4.7070 ; 7.6915)$ \\
$\sigma$ & 0.727 & 0.290 & $(0.3897 ; 1.4691)$ \\
$y^{*}$ & 3.093 & 0.582 & $(1.7374 ; 4.0236)$ \\
\hline
\end{tabular}

more affected by problems with the Pricker. Further, reliability models were individually adjusted, and thereby compared, as described in the next section.

3.2. Preventive Maintenance. In this section, we discuss a parametric approach in order to perform a predictive analysis for the lifetime of the components.

3.2.1. Pricker from Machine A. Table 2 presents a high defect rate after a short repair time as well, compromising the cost of the production. The experiment considered a total period of 30 months, as said before. The operating equipment had three off-seasons; these periods were not included in the dataset. The equipment was only observed during the time of its active operation.

Figure 2 presents the TTT-plot and the survival function fitted by different generalizations of the Weibull distribution.

From the TTT-plot, we observed that the proposed data has unimodal hazard rate, which implies that all the proposed models may be used to describe the proposed dataset. Additionally, the survival function adjusted by the different distributions shows that the proposed models provide a good fit for the proposed data. In order to discriminate the best fit, we considered the results of AIC and AICc (see Table 3).

Among the proposed models, the exponentiated Weibull distribution has superior goodness of fit since the AIC and AICc returned smaller values. Therefore, using the exponentiated Weibull distribution, we computed the maximum likelihood estimates and the predictive value for 25\% (see Table 4). Hereafter, as we considered the quantile function to obtain the predictive value, the confidence intervals (CI) related to this estimate were obtained from bootstrap technique [27]. 

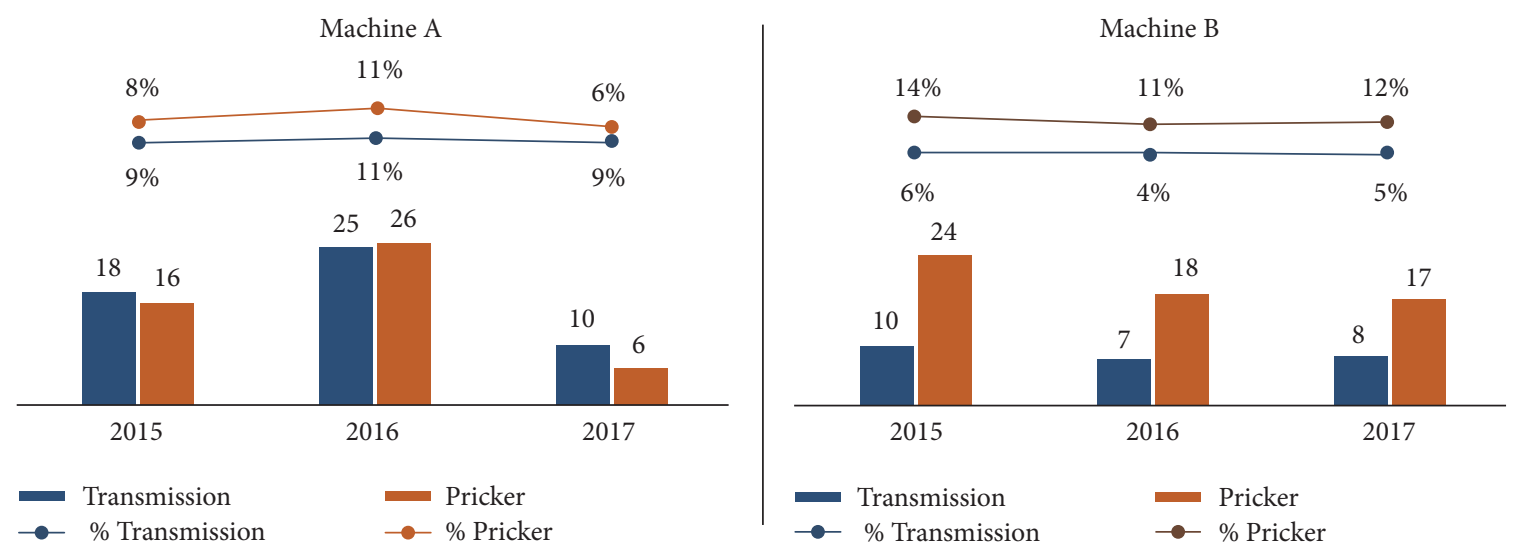

FIgURE 1: Maintenance distribution in each harvester.

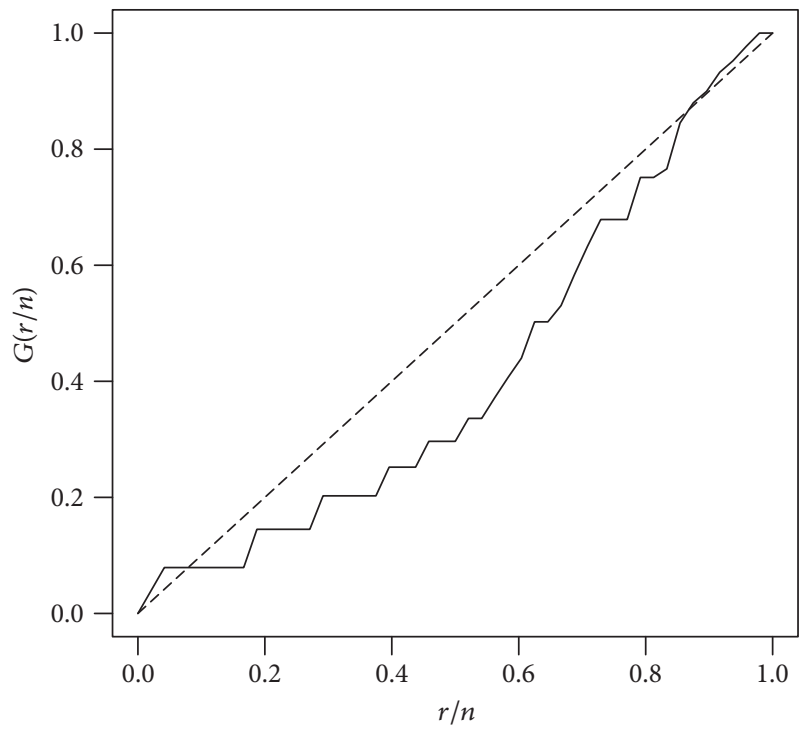

(a)

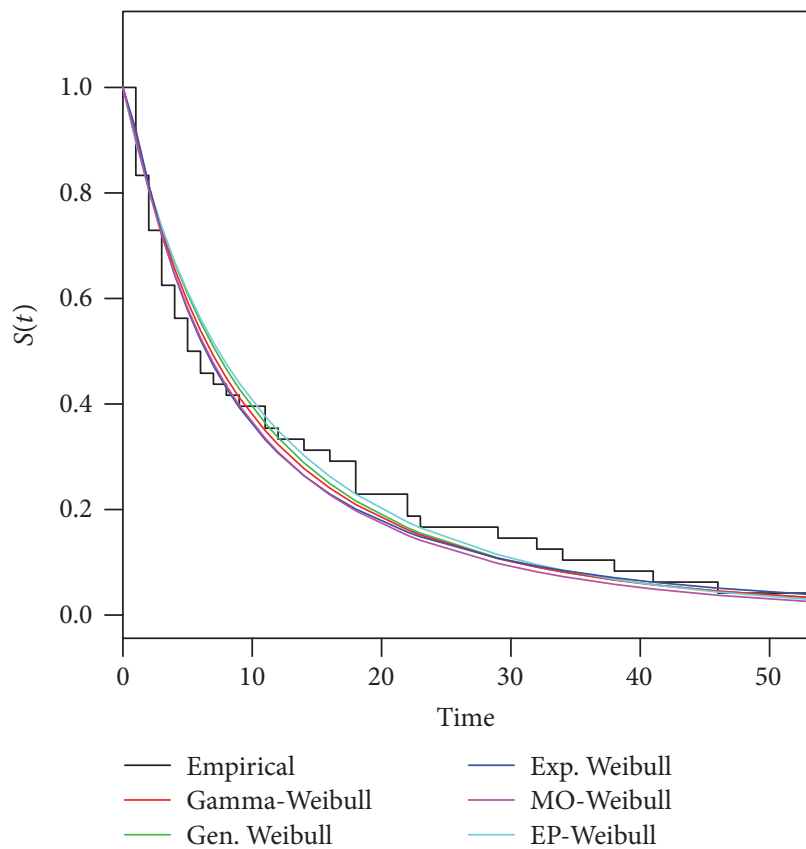

(b)

Figure 2: Pricker A empirical (a) TTT-plot, and (b) contains the fitted survival superimposed to the empirical survival function and the hazard function adjusted by distribution.

From Table 4, we observe that the predictive maintenance should be done in approximately 3 days after the last failure with confidence interval between 2 and 4 days.

3.2.2. Pricker from Machine B. A similar behavior is observed for the Pricker in the machine B, shown in Table 5 presenting a high defect rate as well. The approach was maintained considering only the time during its active operation.

Figure 3 presents the TTT-plot and the survival function fitted by different generalizations of the Weibull distribution, similar to the previous machine.

The TTT-plot shows that the proposed data has unimodal hazard rate, which implies that all the proposed models may be used to describe the dataset. Analogously to the previous
TABLE 5: Dataset related to the sugarcane harvester's Pricker B.

\begin{tabular}{cccccccccccc}
\hline 1 & 1 & 1 & 1 & 1 & 1 & 1 & 1 & 1 & 1 & 1 & 1 \\
2 & 2 & 2 & 3 & 3 & 3 & 3 & 3 & 3 & 4 & 4 & 5 \\
5 & 5 & 5 & 5 & 5 & 5 & 6 & 7 & 7 & 8 & 8 & 8 \\
8 & 8 & 9 & 9 & 11 & 11 & 11 & 11 & 11 & 11 & 12 & 13 \\
14 & 16 & 16 & 21 & 23 & 24 & 27 & 28 & 38 & 43 & 44 & \\
\hline
\end{tabular}

case, the survival function adjusted by the different distributions shows that the proposed models provide a good fit for the proposed data. Therefore, to discriminate the best fit, we considered the results of AIC and AICc (see Table 6).

From the obtained results, we observe that the EW distribution also provided the best fit among the proposed model. 




(a)

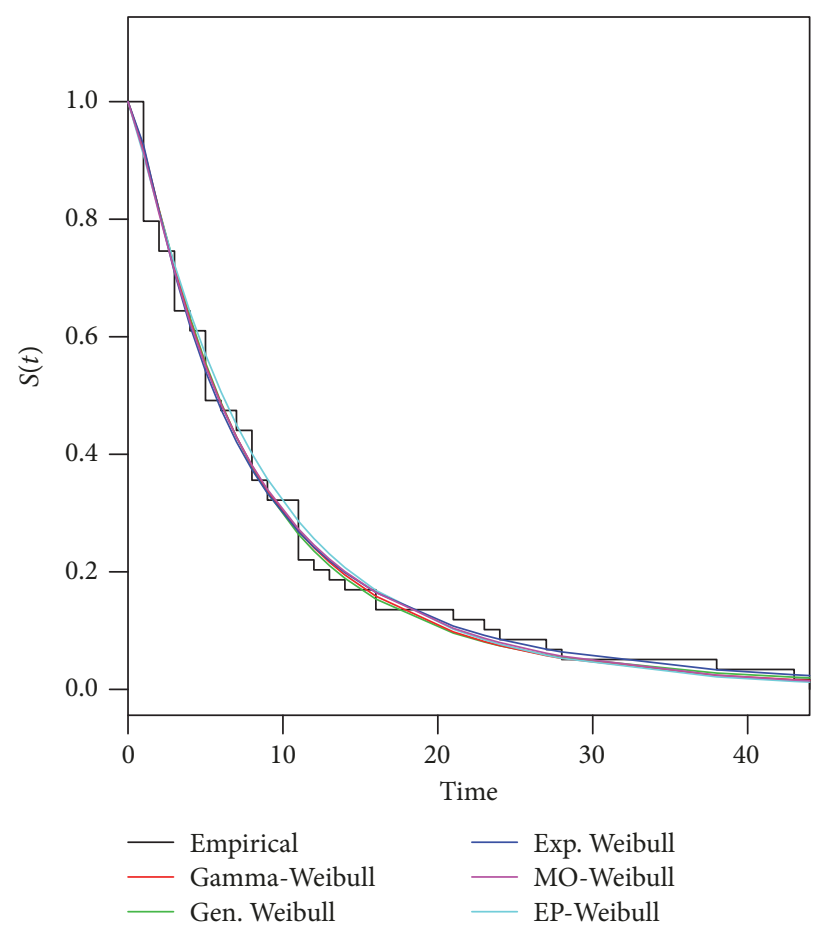

(b)

FIgure 3: Pricker B empirical (a) TTT-plot, and (b) contains the fitted survival superimposed to the empirical survival function and the hazard function adjusted by distribution.

TABLE 6: Results of AIC and AICc criteria and the $p$ value from the KS test for all fitted distributions considering the Pricker B.

\begin{tabular}{lccccc}
\hline Criteria & GG & GW & EW & MOW & EPW \\
\hline AIC & 382.063 & 384.102 & $\mathbf{3 8 1 . 7 9 0}$ & 382.641 & 383.772 \\
AICc & 376.500 & 378.538 & $\mathbf{3 7 6 . 2 2 6}$ & 377.077 & 384.209 \\
KS & 0.3055 & 0.3628 & 0.2737 & 0.3900 & 0.4443 \\
\hline
\end{tabular}

TABLE 7: MLEs, standard deviations, and 95\% confidence intervals for $\alpha, \theta, \sigma$, and $y^{*}$ related to the EW distribution.

\begin{tabular}{lccc}
\hline $\boldsymbol{\theta}$ & MLE & $\mathrm{SD}$ & $\mathrm{CI}_{95 \%}(\boldsymbol{\theta})$ \\
\hline$\alpha$ & 0.457 & 0.050 & $(0.3955 ; 0.5835)$ \\
$\theta$ & 5.434 & 0.763 & $(3.5493 ; 6.8379)$ \\
$\sigma$ & 1.083 & 0.327 & $(0.6879 ; 1.9727)$ \\
$y^{*}$ & 2.497 & 0.459 & $(1.8212 ; 3.5760)$ \\
\hline
\end{tabular}

Furthermore, the maximum likelihood estimates for the EW distribution were computed as well as the predictive value for $25 \%$. Table 7 presents the MLEs, standard deviations, and $95 \%$ confidence intervals for $\alpha, \theta, \sigma$, and $y^{*}$ related to the EW distribution.

Table 7 results suggest that predictive maintenance should be done in approximately 3 days, considering a point estimation, or given a $95 \%$ confidence interval, it would be between 2 to 4 days approximately. Thereby, Pricker among machines showed no difference in performance ever.
TABLE 8: Dataset related to the sugarcane harvester's transmission A.

\begin{tabular}{cccccccccccc}
\hline 1 & 1 & 1 & 1 & 1 & 1 & 1 & 1 & 1 & 1 & 1 & 1 \\
2 & 2 & 2 & 3 & 3 & 3 & 3 & 4 & 5 & 6 & 6 & 6 \\
6 & 7 & 7 & 8 & 8 & 8 & 11 & 11 & 12 & 13 & 13 & 13 \\
14 & 15 & 16 & 17 & 18 & 18 & 19 & 19 & 21 & 22 & 23 & 29 \\
31 & 32 & 34 & 44 & 52 & & & & & & & \\
\hline
\end{tabular}

3.2.3. Transmission from Machine A. Table 8 shows that more than $50 \%$ of the defect rate appears until 8 days right after its repair for the transmission for the machine $\mathrm{A}$.

Figure 4 presents the TTT-plot and the survival function fitted by different generalizations of the Weibull distribution.

As can be seen in the TTT-plot, we observed that the proposed data has also fulfilled the hazard rate shape presupposition. However, from the survival function, there is an indication that the generalized Weibull distribution is not a good candidate to describe the propose data. Table 9 presents the results of AIC and AICc in order to discriminate the best fit.

From Table 9 we can see that the GW distribution has the $p$ value of the KS test smaller than 0.05 ; therefore, it is not a possible candidate to fit the data. Overall, the GG distribution has a better fit, since it has the smaller AIC and AICc. Therefore, we computed the maximum likelihood estimates and the predictive value for $25 \%$ using the GG distribution. Table 10 presents the MLEs, standard deviations, and $95 \%$ 


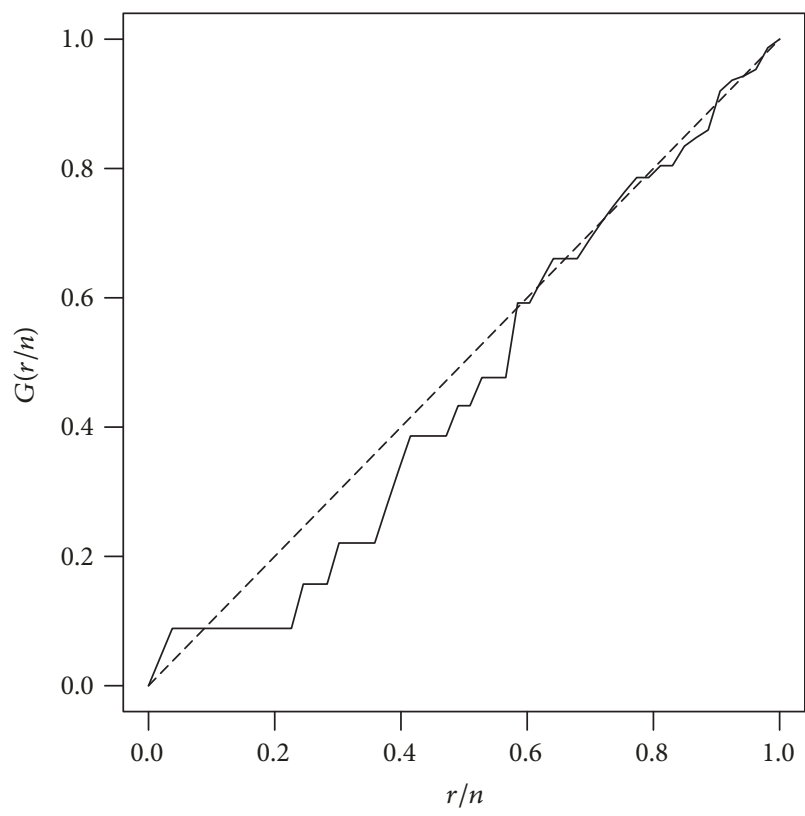

(a)

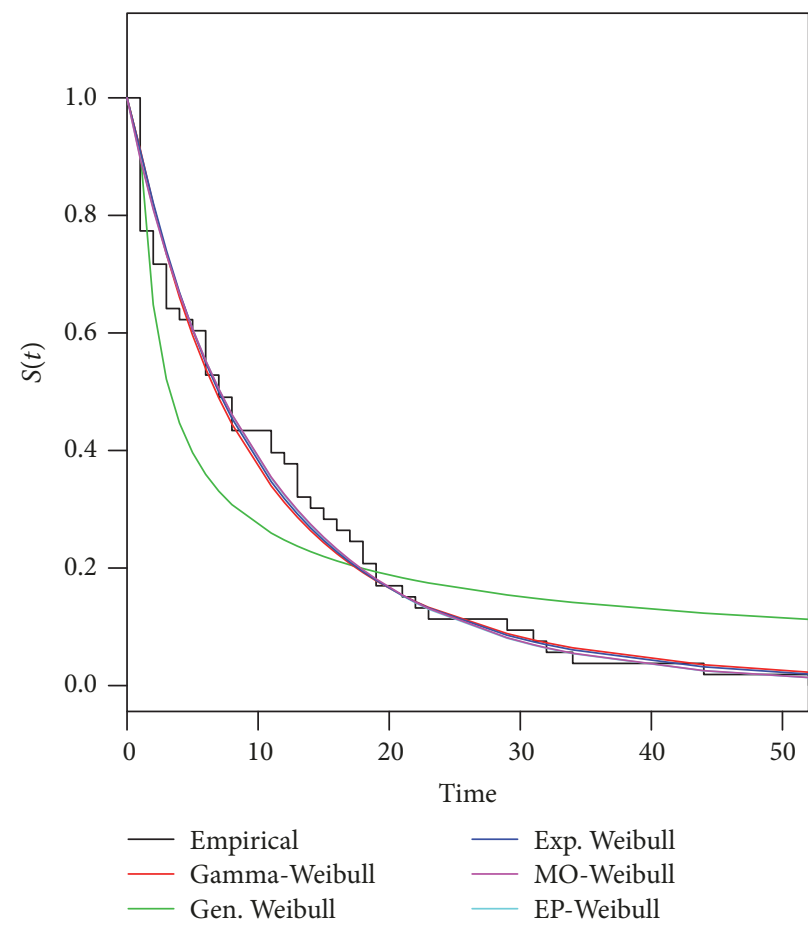

(b)

FIgURE 4: Transmission A empirical (a) TTT-plot, and (b) contains the fitted survival superimposed to the empirical survival function and the hazard function adjusted by distribution.

TABLE 9: Results of AIC and AICc criteria and the $p$ value from the KS test for all fitted distributions considering the Pricker B.

\begin{tabular}{lccccc}
\hline Criteria & GG & GW & EW & MOW & EWP \\
\hline AIC & $\mathbf{3 6 8 . 0 7 4}$ & 381.036 & 368.271 & 368.375 & 368.385 \\
AICc & $\mathbf{3 6 2 . 5 6 3}$ & 375.526 & 362.761 & 362.864 & 368.875 \\
KS & 0.2532 & 0.0035 & 0.2672 & 0.3945 & 0.3738 \\
\hline
\end{tabular}

TABLE 10: MLE, standard deviation, and $95 \%$ confidence intervals for $\phi, \mu, \alpha$, and $y^{*}$ related to the GG distribution.

\begin{tabular}{lccc}
\hline $\boldsymbol{\theta}$ & MLE & $\mathrm{SD}$ & $\mathrm{CI}_{95 \%}(\boldsymbol{\theta})$ \\
\hline$\phi$ & 3.011 & 0.543 & $(1.7396 ; 3.9936)$ \\
$\mu$ & 1.086 & 0.525 & $(0.2389 ; 2.2682)$ \\
$\alpha$ & 0.495 & 0.075 & $(0.4214 ; 0.7124)$ \\
$y^{*}$ & 2.807 & 0.635 & $(1.8487 ; 4.3526)$ \\
\hline
\end{tabular}

confidence intervals for $\phi, \mu, \alpha$, and $y^{*}$ related to the GG distribution.

Table 10 results suggest that predictive maintenance should be done in approximately 3 days, considering a point estimation, or given a $95 \%$ confidence interval, it would be between 2 to 4 days approximately.

3.2.4. Transmission from Machine $B$. Comparing to the other equipment, the transmission from the machine B presented smaller number of occurrence. Table 11 shows the sparsity of
TABLE 11: Dataset related to the sugarcane harvester's transmission B.

\begin{tabular}{lcccccccccc}
\hline 1 & 2 & 3 & 3 & 4 & 5 & 6 & 6 & 7 & 9 & 11 \\
12 & 12 & 18 & 19 & 21 & 23 & 28 & 31 & 31 & 35 & 37 \\
39 & 46 & 61 & & & & & & & & \\
\hline
\end{tabular}

TABLE 12: Results of AIC and AICc criteria and the $p$ value from the KS test for all fitted distributions considering the transmission B.

\begin{tabular}{lccccc}
\hline Criteria & GG & GW & EW & MOW & EWP \\
\hline AIC & 202.220 & 203.201 & 202.833 & 202.368 & $\mathbf{2 0 1 . 9 9 7}$ \\
AICc & 197.363 & 198.344 & 197.975 & 197.511 & $\mathbf{2 0 3 . 1 4 0}$ \\
KS & 0.9382 & 0.7657 & 0.8732 & 0.7710 & 0.9622 \\
\hline
\end{tabular}

the dataset related to the sugarcane harvester's transmission B.

Figure 5 presents the TTT-plot and the survival function fitted by different generalizations of the Weibull distribution, considering the transmission from machine $\mathrm{B}$.

From the TTT-plot, we observed that the proposed data has bathtub shape. Moreover, the adjusted survival functions show that all models are candidates to describe the lifetime of the transmission from the machine $\mathrm{B}$.

Table 12 presents the results of AIC and AICc in order to discriminate the best fit.

As shown in Table 12, the EWP distribution has the minimum AIC and AICc. Therefore, we computed its maximum likelihood estimates and predictive value for $25 \%$, 


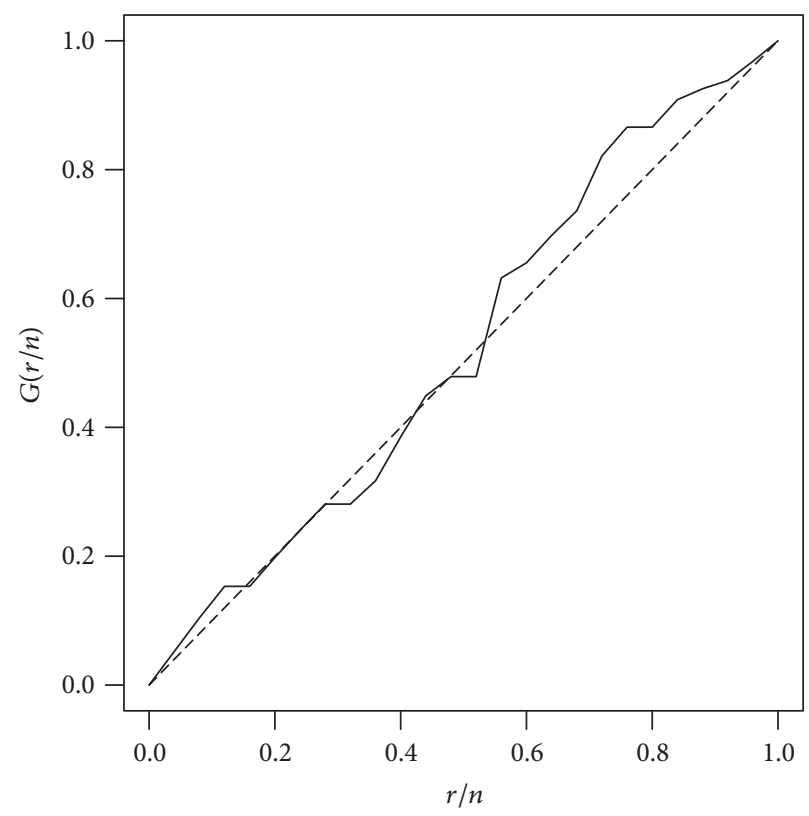

(a)



(b)

FIgURE 5: Transmission B empirical (a) TTT-plot, and (b) contains the fitted survival superimposed to the empirical survival function and the hazard function adjusted by distribution.

TABLE 13: MLEs, standard deviations, and 95\% confidence intervals for $\alpha, \theta, \sigma$, and $y^{*}$ related to the EPW distribution.

\begin{tabular}{lccc}
\hline $\boldsymbol{\theta}$ & MLE & $\mathrm{SD}$ & $\mathrm{CI}_{95 \%}(\boldsymbol{\theta})$ \\
\hline$\phi$ & 0.022 & 0.034 & $(0.0137 ; 0.1350)$ \\
$\lambda$ & -0.572 & 0.541 & $(-1.2492 ; 1.1886)$ \\
$\alpha$ & 1.206 & 0.137 & $(0.7579 ; 1.3705)$ \\
$y^{*}$ & 6.748 & 1.387 & $(3.8716 ; 9.5585)$ \\
\hline
\end{tabular}

respectively. Table 13 presents the MLEs, standard deviations, and $95 \%$ confidence intervals for $\alpha, \theta, \sigma$, and $y^{*}$ related to the EWP distribution.

Table 13 results suggest that predictive maintenance should be done in approximately 7 days, considering a point estimation, or given a $95 \%$ confidence interval, it would be between 4 to 10 days approximately.

\section{Final Remarks}

In this study, we considered different distributions to describe the lifetime of sugarcane harvesting machine components. The harvesters stand out for having a large number of corrective stops, given the functionality in such extreme environmental conditions. However, these harvesters do not have an effective preventive maintenance policy which affects its working time schedule. To overcome this problem, we presented a predictive analysis using probability models based on its percentiles aiming to incorporate intelligence into maintenance planning.

The Weibull distribution is a popular model that can be used to describe a wide range of problems; however, it cannot be used to describe data with nonmonotone hazard rate. Thus, many generalizations of the Weibull distribution have been proposed to overcome this problem. Since the proposed datasets have nonmonotone hazard rate, we considered some flexible generalizations such as the Gamma-Weibull, the generalized Weibull, the exponentiated Weibull, Marshall-Olkin Weibull, and the extended Poisson-Weibull distribution. For the proposed distributions, some mathematical functions were discussed as well as the parameter estimators under the maximum likelihood approach.

The proposed distributions were used to fit the datasets using maximum likelihood estimators. The exponential Weibull presented a superior fit for both machines considering the Pricker component; in these cases, we concluded that a predictive maintenance should be done in approximately 3 days. On the other hand, for the transmission component, the distributions that presented better fit were, respectively, the Gamma-Weibull distribution and the extended PoissonWeibull for machines $\mathrm{A}$ and $\mathrm{B}$, where a predictive maintenance should be done, respectively, in 3 and 7 days after the last failure.

Further work should be considered beyond the adjusted models by including many other generalizations of the Weibull distribution. Also a structure of recurrent event data could be included and its forecast accuracy was analyzed. 
Finally, this approach should be implemented as an applicative, helping the maintenance section in their individualized scheduling distributions.

\section{Abbreviations}

Acronyms

GW: Generalized Weibull

EW: Exponentiated Weibull

MOW: Marshall-Olkin Weibull

EPW: Extended Poisson-Weibull

GG: Generalized gamma

MLE: Maximum likelihood estimator

PDF: Probability density function

CDF: Cumulative distribution function

TTT: Total Time on Test

KS: Kolmogorov-Smirnov

AIC: Akaike Information Criterion

AICC: Corrected Akaike Information Criterion

SD: $\quad$ Standard deviation

CI: Confidence interval.

\section{Notations}

$f(\cdot)$ : Probability density function

$E(\cdot)$ : Mean function

$\operatorname{Var}(\cdot)$ : Variance function

$\gamma(y, x)$ : Lower incomplete gamma function

$\Gamma(y, x)$ : Upper incomplete gamma function

$F(\cdot)$ : Cumulative distribution function

$S(\cdot)$ : Survival function

$h(\cdot)$ : Hazard rate function

$L(\cdot)$ : Likelihood function

$l(\cdot)$ Log-likelihood function

$\psi(\cdot)$ : Digamma function

$\psi^{\prime}(\cdot)$ : Trigamma function

$I(\cdot)$ Expected Fisher information matrix

$\mu$ : $\quad$ Positive parameter

$\phi: \quad$ Positive parameter

$\alpha: \quad$ Positive parameter

$\beta$ : $\quad$ Real parameter

$\lambda: \quad$ Positive parameter

$Q(\cdot)$ : Quantile function

$\Theta: \quad$ Vector of parameters

$H(\cdot)$ : Observed Fisher information matrix

$\mu_{k}: \quad k$ th moment

$G(\cdot): \quad$ TTT-plot

$D_{n}: \quad$ Kolmogorov-Smirnov statistic

$y^{*}: \quad$ Predictive value.

\section{Conflicts of Interest}

No potential conflicts of interest were reported by the authors.

\section{Acknowledgments}

The research was partially supported by CNPq, FAPESP, and CAPES of Brazil.

\section{References}

[1] B. N. S. Company, "Accompanying brazilian safra: Sugarcane," in Harvest 2017/18, vol. 4, pp. 1-57, 2017.

[2] E. Network, "From manual to mechanical harvesting: Reducing environmental impacts and increasing cogeneration potential, 2012".

[3] J. Moubray, Reliability-Centered Maintenance, Industrial Press Inc, 1997.

[4] S. Supsomboon and K. Hongthanapach, "A simulation model for machine efficiency improvement using reliability centered maintenance: Case study of semiconductor factory," Modelling and Simulation in Engineering, vol. 2014, Article ID 956182, pp. $1-9,2014$.

[5] C. Sriram and A. Haghani, "An optimization model for aircraft maintenance scheduling and re-assignment," Transportation Research Part A: Policy and Practice, vol. 37, no. 1, pp. 29-48, 2003.

[6] J. F. Lawless, Statistical Models and Methods for Lifetime Data, vol. 362, John Wiley \& Sons, New York, NY, USA, 1982.

[7] W. Weibull, "Wide applicability," Journal of Applied Mechanics, vol. 103, no. 730, pp. 293-297, 1951.

[8] K. G. Manton and A. I. Yashin, Inequalities of Life: Statistical Analysis And Modeling Perspectives, vol. 145, Human Clocks: The Bio-cultural Meanings of Age 5, 2006.

[9] J. I. McCool, Using the Weibull Distribution: Reliability, Modeling And Inference, vol. 950, John Wiley and Sons, 950, 2012.

[10] D. N. P. Murthy, M. Bulmer, and J. A. Eccleston, "Weibull model selection for reliability modelling," Reliability Engineering \& System Safety, vol. 86, no. 3, pp. 257-267, 2004.

[11] H. Pham and C.-D. Lai, "On recent generalizations of the Weibull distribution," IEEE Transactions on Reliability, vol. 56, no. 3, pp. 454-458, 2007.

[12] C.-D. Lai, "Generalized weibull distributions," in Generalized Weibull Distributions, pp. 23-75, Springer, Heidelberg, Germany, 2014.

[13] M. H. Tahir and G. M. Cordeiro, "Compounding of distributions: a survey and new generalized classes," Journal of Statistical Distributions and Applications, vol. 3, no. 1, 2016.

[14] E. W. Stacy, "A generalization of the gamma distribution," Annals of Mathematical Statistics, vol. 33, pp. 1187-1192, 1962.

[15] G. S. Mudholkar, D. K. Srivastava, and G. D. Kollia, "A generalization of the Weibull distribution with application to the analysis of survival data," Journal of the American Statistical Association, vol. 91, no. 436, pp. 1575-1583, 1996.

[16] G. S. Mudholkar, D. K. Srivastava, and M. Friemer, “The exponentiated weibull family: a reanalysis of the bus-motor-failure data," Technometrics, vol. 37, no. 4, pp. 436-445, 1995.

[17] A. W. Marshall and I. Olkin, "A new method for adding a parameter to a family of distributions with application to the exponential and Weibull families," Biometrika, vol. 84, no. 3, pp. 641652, 1997.

[18] P. L. Ramos, D. K. A. Dey, F. Louzada, and V. H. Lachos, "An extended poisson family of life distribution: A unified approach in competitive and complementary risks," Tech. Rep., University of Connecticut, 2017.

[19] F. Louzada, "Polyhazard models for lifetime data," Biometrics, vol. 55, no. 4, pp. 1281-1285, 1999.

[20] P. L. Ramos, J. A. Achcar, and E. Ramos, "Metodo eficiente para calcular os estimadores de maxima verossimilhanca da distribuicao gama generalizada," Revista Brasileira de Biologia, vol. 32, no. 2, pp. 267-281, 2014. 
[21] P. L. Ramos, J. A. Achcar, F. A. Moala, E. Ramos, and F. Louzada, "Bayesian analysis of the generalized gamma distribution using non-informative priors," Statistics, vol. 51, no. 4, pp. 824-843, 2017.

[22] J. A. Achcar, P. L. Ramos, and E. Z. Martinez, "Some computational aspects to find accurate estimates for the parameters of the generalized gamma distribution," Pesquisa Operacional, vol. 37, no. 2, pp. 365-385, 2017.

[23] A. Choudhury, "A simple derivation of moments of the Exponentiated Weibull distribution," Metrika, vol. 62, no. 1, pp. 17-22, 2005.

[24] G. M. Cordeiro and A. J. Lemonte, "On the marshall-olkin extended weibull distribution," Statistical Papers, vol. 54, no. 2, pp. 1-12, 2013.

[25] F. Hemmati, E. Khorram, and S. Rezakhah, "A new three-parameter ageing distribution," Journal of Statistical Planning and Inference, vol. 141, no. 7, pp. 2266-2275, 2011.

[26] R. E. Barlow and R. A. Campo, "Total time on test processes and applications to failure data analysis," DTIC Document, 1975.

[27] B. Efron and R. J. Tibshirani, An Introduction to the Bootstrap, CRC Press, 1994. 


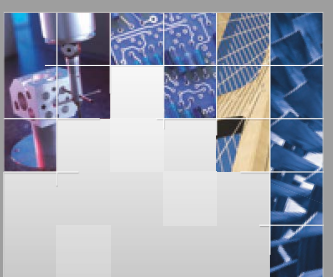

\section{Enfincering}
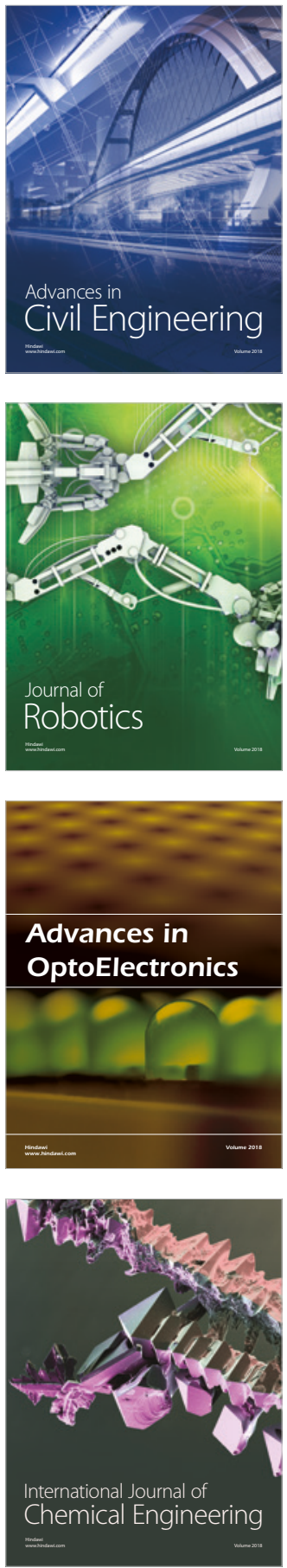

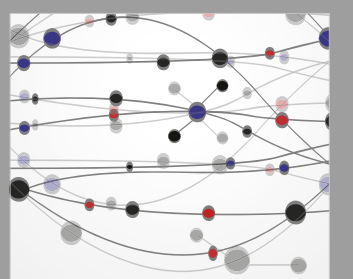

\section{Rotating \\ Machinery}

The Scientific World Journal



Submit your manuscripts at

www.hindawi.com
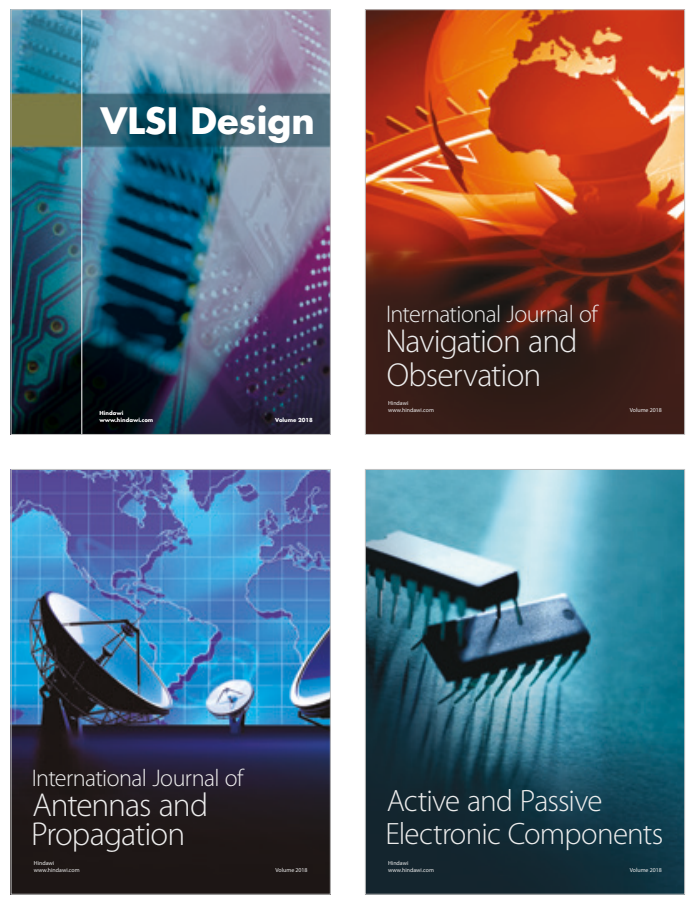
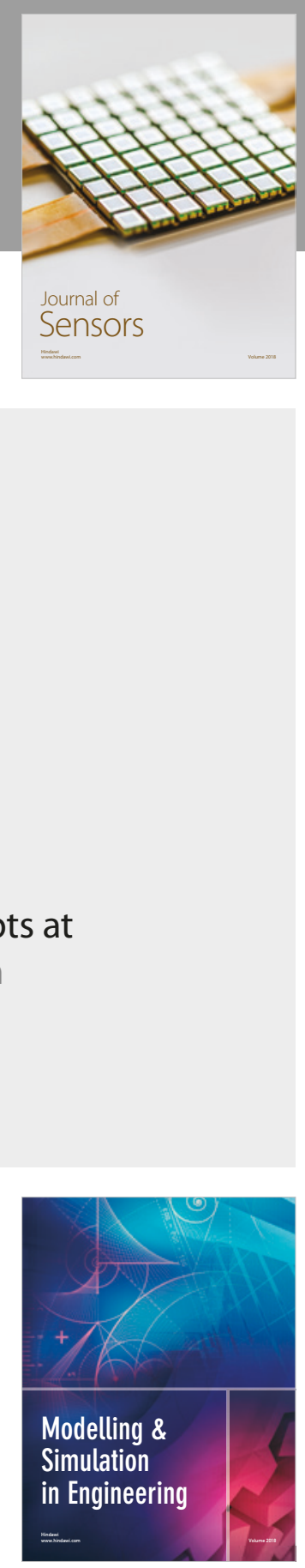

\section{Advances \\ Multimedia}
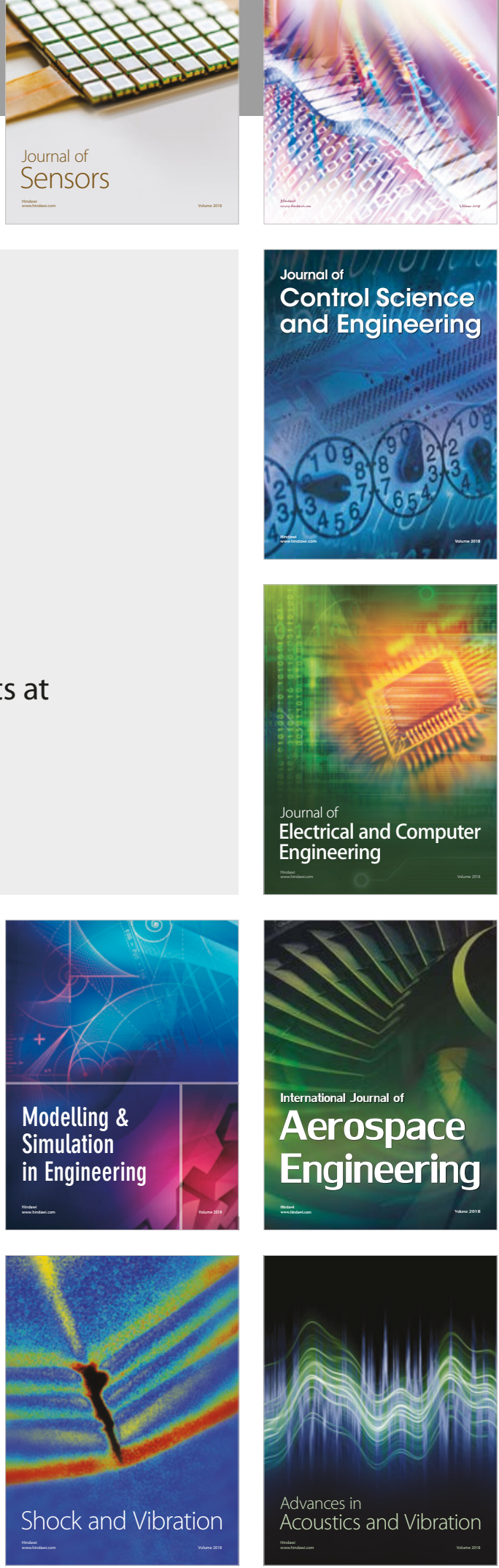